\title{
INVESTIGATION ON THE WAKE FLOW INSTABILITY BEHIND ISOLATED ROUGHNESS ELEMENTS ON THE FOREBODY OF A BLUNT GENERIC REENTRY CAPSULE
}

\author{
A. Theiss and S. Hein \\ German Aerospace Center (DLR) \\ Institute of Aerodynamics and Flow Technology \\ 10 Bunsenstr., Göttingen 37073, Germany
}

\begin{abstract}
Numerical results on modal disturbance growth in the wake flow downstream of a roughness element submerged in the boundary layer of a typical reentry capsule at an angle of attack are presented. Laminar base flow computations were conducted for different roughness heights and planform shapes. The modal instability characteristics of the wake flow were studied by spatial two-dimensional (2D) eigenvalue analysis. For all cases considered, the varicose wake modes are most amplified in terms of maximum $N$-factors, with the cylindrical roughness element being the most effective shape.
\end{abstract}

\section{INTRODUCTION}

In the post Space Shuttle era, manned planetary reentry capabilities rely entirely on capsules. The heat shield of those blunt bodies usually has a spherical-like shape, like the former Apollo capsule or the still operational Soyuz capsule together with its Chinese equivalent Shenzhou. Future manned reentry vehicles will also have a blunt heat shield, as the now being developed Orion Crew Exploration Vehicle (CEV). During the reentry phase, the thermal protection system (TPS) has to withstand very high heat loads. So far, the design philosophy assumed fully turbulent flow in flight at all times, but it is known that over a wide range along the projected return path, the flow is laminar before it transitions to turbulence. The heat flux in the turbulent case can locally be of one order higher than in the laminar regime, which under current design assumptions leads to an overdimensioning of the heat shield concomitant with a reduced payload.

(C) The authors, published by EDP Sciences. This is an Open Access article distributed under the terms of the Creative Commons Attribution License 4.0 (http://creativecommons.org/licenses/by/4.0/). 
In order to reduce the heat shield weight, all flow regimes have to be considered. Therefore, a reliable prediction of the transition onset location is crucial. Due to a lack of appreciation and understanding of the physics of transition on such blunt vehicles, the transition onset location had usually been correlated to some boundary layer values such as Reynolds number based on displacement or momentum thickness. A detailed overview of wind tunnel measurements for two capsules with different heat shield shapes (spherical heat shield for CEV and sphere-cone shape for the Mars Science Laboratory (MSL)), with empirical correlations for transition onset, is given by Hollis [1]. For the design of future returning space vehicles, those correlations should be replaced by the methods based on stability theory and transient growth considerations as demanded by Reshotko [2]. So far, stability analyses have only been performed for the MSL capsule, where transition was dominated by first-mode and cross-flow instabilities [3,4] and recently by Theiss et al. [5] for an Apollo-shaped capsule. Because of a strong favourable pressure gradient, the boundary layer in the latter investigation did not feature any amplified modal disturbances at similar freestream conditions to [4].

There exist several sources of instability and mechanisms leading to turbulence which are specific to hypersonic flows over blunt capsules. One of them is outgassing from an ablative heat shield. Li et al. [6] demonstrated the destabilizing effect of ablation-induced outgassing on a boundary flow over a hemispherical capsule which otherwise did not support amplification of instability modes. Ablation of the TPS leads to distributed surface roughness which, in turn, causes an earlier boundary-layer transition compared to the smooth wall case (see [7]). Discrete surface inhomogeneities such as attachment-point cavities, compression pads, gaps or steps between heat shield tiles or unevenly ablated TPS material can also lead to premature laminar-turbulent transition downstream of the protuberance element (the type of transition investigated in the present study) or even bypass transition if the roughness height/cavity depth is sufficiently large. Amar et al. [8] demonstrated the effect of isolated protuberances on the laminar to turbulent transition on the CEV capsule in wind tunnel experiments. Transition to turbulence was induced by a trip array of three diamond-shaped discrete roughness elements on the leeward side, but not on the windside even for the largest $(k / \delta \approx 1)$ element at the highest wind tunnel Reynolds number. Chang et al. [9] numerically investigated the effect of surface irregularities in the form of a protuberance and cavity on the CEV surface by means of direct numerical simulation (DNS). The authors showed that the investigated cylindrical protrusion element is more effective to trigger transition than a cavity at identical roughness Reynolds number $\mathrm{Re}_{k k}$ and Reynolds number values. An extensive summary on roughness related transition in hypersonic boundary layers on blunt bodies is given by Schneider [10].

The mechanisms leading to transition behind a three-dimensional (3D) roughness are only partly understood, despite the substantial number of experimental 
and numerical studies on this topic. Similar to the smooth-wall case without surface inhomogeneities, the onset of roughness-induced transition is still predicted based on empirical correlations (e.g., $\mathrm{Re}_{k k}$ ) [11]. A physics-based prediction of roughness-induced transition is not yet existent, but extensive DNS simulations and results from 2D linear eigenvalue solutions have considerably increased the knowledge in the recent years. The wake flow behind an isolated roughness element has a strong inhomogeneous character in both wall-normal and spanwise direction. Thus, the conventional linear stability analysis, which is based on the assumption of inhomogeneity in just one spatial direction, is not appropriate to analyze the stability characteristics for this kind of flow and a $2 \mathrm{D}$ eigenvalue problem has to be solved instead. Groskopf and coauthors [12] performed temporal $2 \mathrm{D}$ eigenvalue analyses on the instability of an isolated $3 \mathrm{D}$ roughness element in a Mach 4.8 boundary layer and reported a very good agreement to results from a DNS. Recent measurements via hot-wire anemometry by Kegerise et al. [13] behind a diamond element in a Mach 3.5 flat plate boundary layer showed a close agreement of spatial distribution of measured disturbance amplitude with the spatial distribution of the computed eigenfunction for the most dominant wake mode, further proving the applicability of $2 \mathrm{D}$ instability theory to wake flows. So far, $2 \mathrm{D}$ instability theory has only been used to calculate the instability characteristics behind discrete roughness elements on a flat plate, but not yet for an isolated roughness element located on the heat shield of a reentry capsule.

In this work, the results from laminar base flow computations are reported for the wake flow behind a 3D roughness element submerged in the leeward side boundary layer of an Apollo-shaped capsule at an angle of attack AOA $=24^{\circ}$ for freestream conditions taken from a measurement campaign at the Hypersonic Ludwieg tube Braunschweig (HLB). The effect of different roughness heights on the modal instability characteristics behind a cylindrical roughness element are investigated with the help of spatial $2 \mathrm{D}$ instability theory. Instability results for a cuboidal and diamond element will also be shown in order to examine the influence of different roughness shapes on the growth rates and eigenfunctions for the dominant wake modes.

\section{GOVERNING EQUATIONS}

\subsection{Navier-Stokes Equations}

In the present work, a system of nonlinear partial differential equations governing the motion of a Newtonian fluid (Navier-Stokes equations) is solved. The equations for flow of a perfect gas with density $\rho$, pressure $p$, internal energy $e$, and velocity component $u_{i}$ in the $i$ th coordinate direction $(i=1,2,3)$ are written in conservation laws of mass, momentum, and energy: 


$$
\begin{array}{r}
\frac{\partial \rho}{\partial t}+\frac{\partial\left(\rho u_{j}\right)}{\partial x_{j}}=0 \\
\frac{\partial\left(\rho u_{i}\right)}{\partial t}+\frac{\partial\left(\rho u_{i} u_{j}\right)}{\partial x_{j}}+\frac{\partial p}{\partial x_{i}}-\frac{\partial \tau_{i j}}{\partial x_{j}}=0 \\
\frac{\partial(\rho E)}{\partial t}+\frac{\partial\left(\rho E u_{i}+p u_{i}\right)}{\partial x_{i}}+\frac{\partial q_{i}}{\partial x_{i}}-\frac{\partial\left(\tau_{i j} u_{i}\right)}{\partial x_{j}}=0
\end{array}
$$

where $E=e+u_{i} u_{i} / 2$ and $t$ is the time. The symmetric viscous stress tensor $\tau_{i j}$ is defined by

$$
\tau_{i j}=\mu\left(\frac{\partial u_{i}}{\partial x_{j}}+\frac{\partial u_{j}}{\partial x_{i}}-\frac{2}{3} \frac{\partial u_{k}}{\partial x_{k}} \delta_{i j}\right)
$$

where $\mu$ is the dynamic viscosity and $\delta_{i j}$ is the Kronecker delta defined by

$$
\delta_{i j}= \begin{cases}1 & \text { for } i=j \\ 0 & \text { for } i \neq j\end{cases}
$$

Closure of the equation is obtained through the perfect gas law together with the Fourier's law of heat conduction, given, respectively, by

$$
p=\rho R T ; \quad q_{j}=-\kappa \frac{\partial T}{\partial x_{j}}
$$

where $T$ is the temperature; $R$ is the specific gas constant; and $\kappa$ is the thermal conductivity. A two-part Sutherland's law (one for low temperatures ( $T$ $<120 \mathrm{~K}$ ) and the standard law for $T \geq 120 \mathrm{~K}$ ) is used for the calculation of the dynamic viscosity. The thermal conductivity is related to $\mu$ through the relation $\kappa=c_{p} \mu / \operatorname{Pr}$ where $c_{p}$ is the specific heat at constant pressure and $\operatorname{Pr}$ is the molecular Prandtl number (in this work, Pr is assumed to be 0.72).

\subsection{Spatial Two-Dimensional Stability Equations}

The linear spatial 2D stability solver is based on the nondimensional version of the compressible Navier-Stokes equations (1)-(3) and is formulated in primitive variables and uses general, orthogonal curvilinear coordinates. Analogue to the ansatz of classical linear stability theory, all flow quantities $\mathbf{q}=[u, v, w, T, \rho]^{\mathrm{T}}$ are split into their steady primary state $\overline{\mathbf{q}}$ and unsteady perturbation part $\tilde{\mathbf{q}}$ :

$$
\mathbf{q}(x, y, z, t)=\overline{\mathbf{q}}(y, z)+\epsilon \tilde{\mathbf{q}}(x, y, z, t), \quad \epsilon \ll 1,
$$

whereby the primary state $\overline{\mathbf{q}}$ and the total flow field $\mathbf{q}$ are assumed to satisfy the Navier-Stokes equations. However, two spatial inhomogeneous directions have to be resolved simultaneously on a plane (wall normal $y$ and spanwise $z$ direction), 
whereas spatial homogeneity in the third direction is assumed (streamwise $x$ direction). The infinitesimal $3 \mathrm{D}$ perturbations $\epsilon \tilde{\mathbf{q}}$ are inhomogeneous in $y$ and $z$ and periodic in $x$. After applying the modal perturbation ansatz, $\tilde{\mathbf{q}}$ can be written as

$$
\tilde{\mathbf{q}}(x, y, z, t)=\hat{\mathbf{q}}(y, z) \Theta(x, t)+\text { c.c. }
$$

where $\hat{\mathbf{q}}$ is the vector of the $2 \mathrm{D}$ complex amplitude functions in a plane perpendicular to the $x$-axis;

$$
\Theta=\exp [i(\alpha x-\omega t)]
$$

is the complex phase function; and c.c. denotes the complex conjugate. Substituting the perturbed state (Eq. (4)) into the Navier-Stokes equations (1)-(3), assuming local parallel flow, subtracting out the $\mathcal{O}(1)$ base flow terms, and neglecting the nonlinear terms, which are one order of magnitude smaller than the other ones $\left(\mathcal{O}\left(\epsilon^{2}\right)\right)$, leads to the local linear stability equations. They can be written in the form:

$$
\mathbf{A}_{\mathbf{0}} \hat{\mathbf{q}}+\mathbf{A}_{\mathbf{y}} \frac{\partial \hat{\mathbf{q}}}{\partial y}+\mathbf{A}_{\mathbf{y y}} \frac{\partial^{2} \hat{\mathbf{q}}}{\partial y^{2}}+\mathbf{A}_{\mathbf{z}} \frac{\partial \hat{\mathbf{q}}}{\partial z}+\mathbf{A}_{\mathbf{z z}} \frac{\partial^{2} \hat{\mathbf{q}}}{\partial z^{2}}+\mathbf{A}_{\mathbf{y z}} \frac{\partial^{2} \hat{\mathbf{q}}}{\partial y \partial z}=0 .
$$

The streamwise wavenumber $\alpha$ as well as the circular frequency $\omega$ can both be complex. In this work, the spatial framework has been used; therefore, $\omega$ is real and is related with the circular frequency of an eigenmode $\tilde{\mathbf{q}}$, while $\alpha$ is a complex eigenvalue and its associated eigenvectors $\hat{\mathbf{q}}$ are to be sought. The real part $\alpha_{r}=\mathfrak{R}\{\alpha\}$ is related with the wavenumber of $\tilde{\mathbf{q}}$ along the homogeneous direction $x$ with periodicity length $L_{x}=2 \pi / \alpha_{r}$, whereas the imaginary part, $\alpha_{i}=\Im\{\alpha\}$, is its growth/damping rate. A negative value of $\alpha_{i}$ implies spatial growth of the eigenmode, while a positive value denotes spatial decay of $\tilde{\mathbf{q}}$. After discretization, Eq. (5) can be formed into a 2D Generalized Eigenvalue Problem (GEVP), but the resulting system is nonlinear in eigenvalue $\alpha$. In order to convert the system into a linear eigenvalue problem, the companion matrix method [14] is used, where an auxiliary vector is defined,

$$
\hat{\mathbf{q}}^{+}=[\hat{u}, \hat{v}, \hat{w}, \hat{T}, \hat{\rho}, \alpha \hat{u}, \alpha \hat{v}, \alpha \hat{w}, \alpha \hat{T}]^{\mathrm{T}} .
$$

The resulting $2 \mathrm{D}$ GEVP is

$$
\mathcal{A} \hat{\mathbf{q}}^{+}=\alpha \mathcal{B} \hat{\mathbf{q}}^{+}
$$

\section{NUMERICAL METHODS}

\subsection{Laminar Base Flow Computation Without Roughness Element}

The geometry studied here corresponds to an Apollo-shaped capsule with a spherical forebody (diameter of $170 \mathrm{~mm}$ ) at $\mathrm{AOA}=24^{\circ}$. The laminar base 
Table 1 Overview of investigated freestream condition

\begin{tabular}{cccc}
\hline $\mathrm{Re} / l, 1 / \mathrm{m}$ & $\mathrm{Ma}$ & $T, \mathrm{~K}$ & $p, \mathrm{~Pa}$ \\
\hline $10 \cdot 10^{6}$ & 5.9 & 59.0 & 764.9 \\
\hline
\end{tabular}

flow over the blunt capsule was computed on a block-structured grid using the 3D, finitevolume, compressible Navier-Stokes flow solver FLOWer [15]. For the smooth wall simulations, a second-order AUSMDV upwind scheme was used for the flux discretization and an explicit 5-stage Runge-Kutta time stepping scheme was chosen for the time discretization. To achieve better convergence behavior, the capsule shoulder was flagged turbulent using the one-equation turbulence model of Spalart and Allmaras. Details on the used singularity-free mesh are given in [5]. The computational fluid dynamics (CFD) freestream values were determined from an HLB run condition, which are summarized in Table 1. Due to the short operating times of the windtunnel, a uniform isothermal wall boundary condition was imposed at a temperature of $T=295 \mathrm{~K}$.

\subsection{Laminar Base Flow Computation with Roughness Element}

In this work, the wake flow behind an isolated roughness element located in the leeward symmetry plane of the capsule forebody is investigated. Figure 1 provides a schematic overview of the three different planform shapes used in this study, whereas only half of the element was simulated within a truncated computational domain (shown in Fig. 2), exploiting the azimuthal symmetry of the flow field. The approach of using a domain within a domain significantly reduces the overall grid counts for the laminar base flow generation. The truncated domain is located below the bow shock; so, a second-order central scheme was chosen for the spatial discretization in order to speed up the simulation. The numerical boundary conditions imposed were those as depicted in Fig. 2. Converged base flow data without the protuberance element on the capsule are specified on all sides via a Dirichlet boundary condition, except for the wall, the outflow region, and the capsule zero meridian, where the no-slip, supersonic outflow, and symmetry condition were set, respectively. The suitability of the Dirichlet boundary condition has been checked for an adiabatic smooth wall case, where, based on a converged mean flow solution, a reduced domain was extracted and the same boundary conditions as in Fig. 2 were imposed. The flow field within the boundaries was initialized with zeros. The profiles of the converged flow with the Dirichlet boundary condition were in excellent agreement with the original solution as shown in Fig. 3. In order to avoid any influence of the Dirichlet boundary condition on the flow around the roughness element, the domain boundaries were placed far away from the protuberance element. The largest dimensions of the disturbance element studied in this work are $k=150 \mu \mathrm{m}$ and $d=1 \mathrm{~mm}$, leading to a minimal distance from the front, lateral, and upper Dirichlet surfaces of about $46 d, 56 d$, and $133 k$, respectively. 


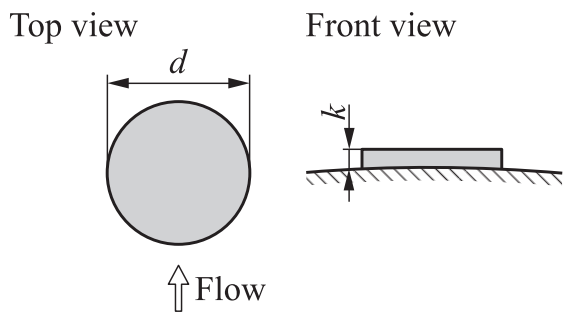

(a)

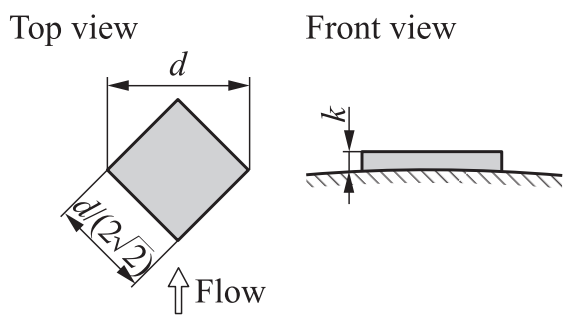

(b)

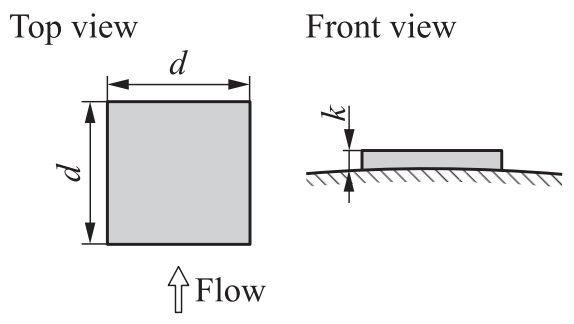

(c)

Figure 1 Schematic of investigated isolated roughness elements with different planform shapes: (a) cylindrical roughness element; (b) diamond; and (c) cuboidal roughness element. Due to the spanwise symmetry of the flow field, only the right half of the element was used in the computations
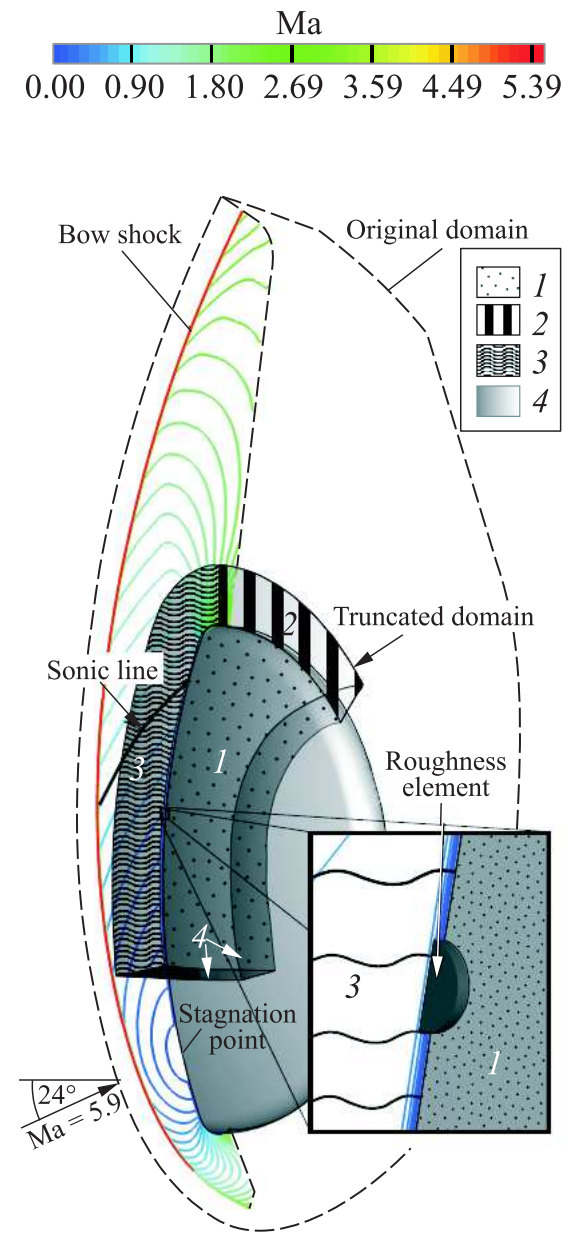

Figure 2 Scheme of the truncated domain used for the roughness wake flow simulations with the specified boundary conditions: 1 - no slip; 2 - supersonic outflow; 3 - symmetry; and 4-Dirichlet. The dashed line indicates the size of the original domain used for the smooth wall configuration at $\mathrm{AOA}=24^{\circ}$ 


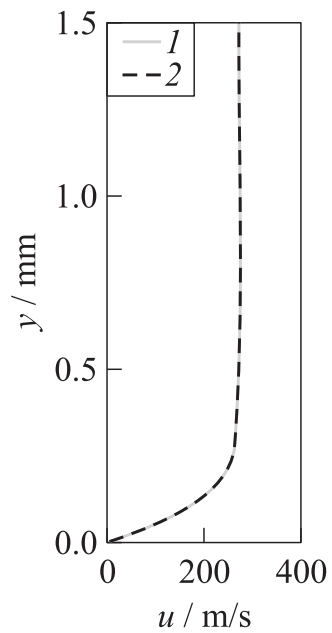

(a)

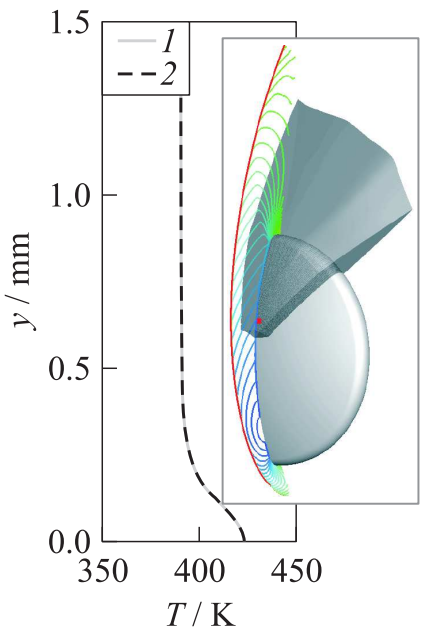

(b)

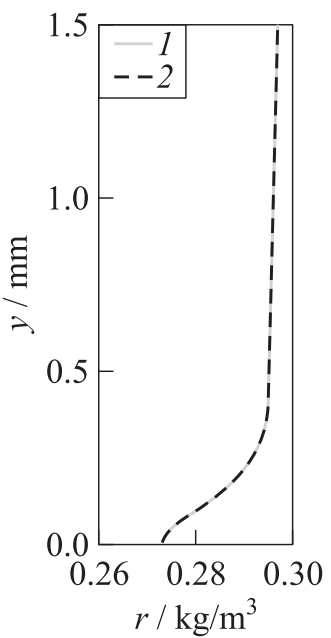

(c)

Figure 3 Comparison of boundary layer profiles computed for the smooth capsule in the original (1) and reduced (2) domains. The boundary layer profiles are extracted at the position marked with the cross within the dark grey colored reduced domain

Block-structured grids are used to discretize the flow field around the sharp edged roughness elements. Mesh generation was accomplished with the freeware program MegaCads, which was developed by DLR. Depending on the planform shape of the disturbance element, different grid topologies were used. However, all grids have about the same number of points behind the roughness element in streamwise, spanwise, and wall normal direction with about 1100, 260, and 300 points, respectively. The total grid count sums up to about $100 \cdot 10^{6}-150$. $10^{6}$ points depending on the topology. To maintain a reasonable computational effort, clustering towards the element in all directions was used. A preliminary grid convergence study has been conducted by increasing the points in streamwise direction by $36 \%$ for the cuboidal element. Figure $4 a$ shows the streak amplitude behind the roughness element computed from the base flows obtained on the coarse and finer grid. The streak amplitude is defined as

$$
A_{\mathrm{st}}=\frac{1}{2}\left[\max _{y, z}\left(u-u_{b}\right)-\min _{y, z}\left(u-u_{b}\right)\right]
$$

where $u$ is the streamwise velocity and $u_{b}$ is the undisturbed laminar base flow. In addition, Fig. $4 b$ depicts the spatial growth rate along the surface arc length $s$ for the dominant symmetric mode at one frequency (a more detailed analysis on the stability characteristics will be given in subsection 5.2). The lines for the 


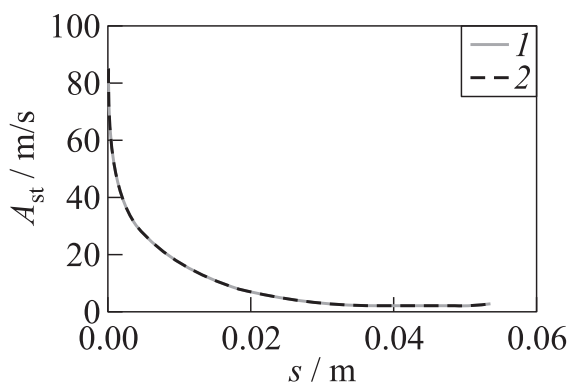

(a)

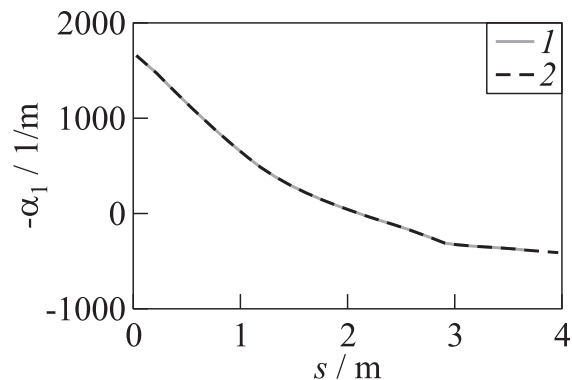

(b)

Figure 4 Streak amplitude vs. surface arc length computed on coarse (1) and finer (2) grids with $36 \%$ more points in streamwise direction $(a)$ and the spatial growth rate vs. surface arc length for the dominant symmetric mode at $f=50 \mathrm{kHz}(b)$. The position $s=0$ represents the trailing edge of the cuboidal element

streak amplitude as well as the lines for the spatial growth rate coincide for both plots. Hence, all simulations were performed with the coarser grid.

\subsection{Two-Dimensional Eigenvalue Computations}

To solve the GEVP (Eq. (6)), adequate boundary conditions should be specified for the disturbance variables. The no-slip condition is imposed at the wall $(y=0)$ by setting the velocity perturbations to zero via a Dirichlet boundary condition. The same condition is also used for the temperature term, whereas no boundary condition needs to be specified for the density fluctuation, because the linearized continuity equation is satisfied at the wall. In wall normal direction, the perturbations are forced to decay by imposing a homogeneous Dirichlet condition at the upper boundary, which is placed far away from the region of interest to avoid reflections. In accordance to the laminar base flow computation, the symmetry at the capsule zero meridian $(z=0)$ is exploited by imposing symmetric and antisymmetric boundary conditions to reduce the computational effort. All perturbations are set to zero for the antisymmetric conditions, except for the azimuthal velocity component where the gradient in spanwise direction is zero. In case of the symmetric boundary condition, the aforementioned specifications are inverted. Corresponding Dirichlet conditions are set on the opposing side.

The matrices $\mathcal{A}$ and $\mathcal{B}$ of the GEVP (Eq. (6)) have a leading dimension of $\mathcal{O}\left(10^{5}\right)$; thus, an efficient assembling and storage implementation is required. The spatial 2D eigenvalue solver forms the matrices in parallel (parallelized via Message Passing Interface (MPI)), using the compressed sparse row storage format from the Portable, Extensible Toolkit for Scientific Computation (PETSc) library [16]. The eigenvalue problem (Eq. (6)) is solved with the help of the 
Scalable Library for Eigenvalue Problem Computations (SLEPc), which is built on top of PETSc, providing all the functionality necessary for the solution of eigenvalue problems [17]. The code is in principle capable of calculating the full eigenvalue spectrum by using the classical QZ method, but this is only workable for small-size problems. For the large-scale eigenvalue problem considered in this work, an iterative projection method is chosen, which only provides a few eigenvalues in the vicinity of a specified estimate, but substantially reduces the needed computational time. Several iterative eigenvalue solvers are available within SLEPc and access is provided to additional software packages. The results presented in the next sections are computed with the implicitly restarted Arnoldi method implemented in the parallel version of the ARPACK $[18,19]$ library, which is interfaced from SLEPc. The method is well suited to extract eigenvalues at the boundaries of the spectra. However, the eigenvalues of interest could be the interior ones and not the ones of the largest/smallest real or imaginary part. Therefore, the shift-and-invert strategy is used and instead of Eq. (6), the following shifted problem is solved:

$$
\mathcal{C} \hat{\mathbf{q}}^{+}=\nu \hat{\mathbf{q}}^{+}, \quad \mathcal{C}=(\mathcal{A}-\sigma \mathcal{B})^{-1} \mathcal{B}, \quad \nu=\frac{1}{\alpha-\sigma}
$$

where $\sigma$ is the shift value in whose vicinity the desired eigenvalues are to be sought. The large matrix inversion involved in Eq. (8) is performed with the MUltifrontal Massively Parallel sparse direct Solver (MUMPS) [20], which is interfaced from PETSc.

The wall-normal and spanwise directions are discretized with stable highorder finite differences schemes of order $q$ (FD- $q$ ), developed by Hermanns and Hernández [21]. The method is based on piecewise polynomial interpolation of degree $q \leq I$ on a nonuniform grid point distribution, where $I+1$ is the number of total grid points. The chosen nonuniform node distribution minimizes the error of the piecewise polynomial interpolation and in case of $q=I$, the resulting finite difference schemes are equivalent to the Chebyshev collocation methods. Paredes et al. [22] showed that this finite difference method outperforms spectral collocation methods for stability analysis calculations and is the best compromise in terms of accuracy and computational efficiency, while recovering spectral-like accuracy. In the present study, the eighth-order scheme, FD- $q 8$, has been chosen and grid convergence was checked by increasing the number of discretization nodes in spanwise and wall-normal direction, respectively. Figure 5 shows the integrated growth rate in terms of an $N$-factor for the dominant symmetric mode behind the cuboidal roughness element with various grid resolutions. The integrated amplification rate, $N$-factor, is defined as follows:

$$
N=\int_{s_{0}}^{s}-\alpha_{i} d s .
$$




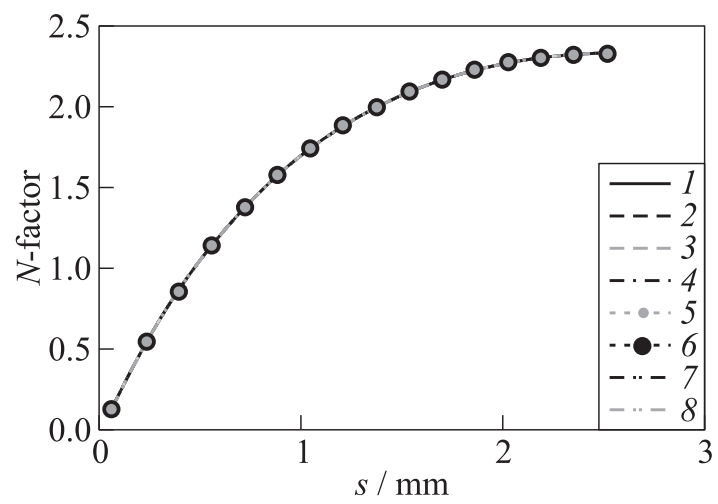

Figure $5 \mathrm{~N}$-factor vs. surface arc length computed for the dominant symmetric mode with $f=90 \mathrm{kHz}$ at various grid resolutions in spanwise and wall-normal directions (pts- $z \times$ pts- $y$ ) behind the cuboidal element: $1-123 \times 101 ; 2-153 \times 101 ; 3-$ $183 \times 101 ; 4-201 \times 101 ; 5-201 \times 121 ; 6-201 \times 141 ; 7-201 \times 161$; and $8-$ $201 \times 181$. The discretization scheme is FD- $q 8$

Here, $s_{0}$ denotes the streamwise position where first disturbances start to grow at a given frequency. Even though the growth rate slightly varies with the grid resolution, the impact on the $N$-factor is negligible and, therefore, all subsequent $2 \mathrm{D}$ spatial eigenvalue computations were performed with 123 points in spanwise and 101 points in wall-normal direction.

The interval of the nonuniform grid point distribution $(\eta \in[-1,1])$ usually does not coincide with the physical domain of the problem studied (here, $\xi$ $\in\left[0, \xi_{\infty}\right]$ with $\left.\xi \hat{=} y, z\right)$; therefore, appropriate mappings need to be applied. In this work, the same mapping function is chosen to cluster the points in the region of the roughness element wall normal extension and towards the symmetry plane:

$$
\xi_{j}=l \frac{1-\eta_{j}}{1+k+\eta_{j}}, \quad k=\frac{2 l}{\xi_{\infty}}, \quad l=\frac{\xi_{\infty} \xi_{h}}{\xi_{\infty}-2 \xi_{h}} .
$$

Here, $\xi_{\infty}$ denotes the location where the domain is truncated and $\xi_{h}$ represents the position where the number of discretization points is split into two halves.

\section{VALIDATION}

The eigenvalue spectrum of a wake flow plane behind a rectangular roughness element submerged into a $\mathrm{Ma}=2.5$ flat plate boundary layer is investigated in order to validate the spatial $2 \mathrm{D}$ eigenvalue solver used in this work, based on the 


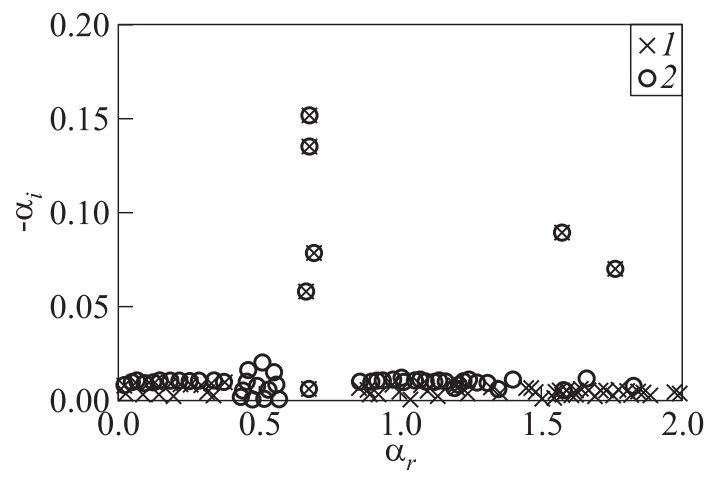

Figure 6 Spatial eigenspectrum behind a rectangular roughness element submerged in a $\mathrm{Ma}=2.5$ flat plate boundary layer. The spectrum is obtained for the conditions given in [24] for $F=0.08$ at $x=93.66$ : 1 - present results; and 2 - De Tullio et al. [24]

results from a different solver, which has been extensively validated $[22,23]$. Details on the base flow computations, as well as on the instability characteristics of the wake modes, studied by DNS and an instability solver, are given in [24]. Figure 6 depicts the spatial eigenspectrum obtained for $F=0.08$ at $x=93.66$. $F$ is the dimensionless frequency used in that study and is defined as $F=f \delta_{1} / u_{\infty}$, where $f$ is the dimensional frequency and $\delta_{1}$ is the displacement thickness at the beginning of the computational domain. For the spectrum shown in Fig. 6, De Tullio and coauthors [24] discretized the whole spanwise extend of the computational domain with standard finite difference schemes and imposed periodic boundary conditions. The wall-normal direction was discretized using the FD- $q$ method. In the current study, the eighth-order, FD- $q 8$, scheme was chosen for both spatial directions and discretized only half of the plane in spanwise direction and symmetric and antisymmetric boundary conditions were imposed at the roughness mid-plane to reduce the computational cost of the simulation and to check the proper implementation of the boundary conditions. The growth rates of the amplified discrete eigenmodes are in excellent agreement with the corresponding published results.

\section{RESULTS}

In the following, results will be presented for all flow cases summarized in Table 2. At first, findings will be shown for the effect of different roughness heights on the laminar base flow and stability properties. In the subsequent subsection the analysis will be extended to different roughness planform shapes. 
Table 2 List of flow cases investigated for the current study

\begin{tabular}{ccccccccc}
\hline Case & Shape & $k, \mu \mathrm{m}$ & $d, \mathrm{~mm}$ & $k / d$ & $k / \delta$ & $k / \delta_{1}$ & $\mathrm{Re}_{k k}$ & $\mathrm{Re}_{k k^{*}}$ \\
\hline 1 & $\bullet$ & 75 & 1 & 0.075 & 0.10 & 0.70 & 124 & 138 \\
2 & $\bullet$ & 105 & 1 & 0.105 & 0.14 & 0.97 & 206 & 237 \\
3 & $\bullet$ & 130 & 1 & 0.130 & 0.17 & 1.21 & 278 & 327 \\
4 & $\bullet$ & 150 & 1 & 0.150 & 0.20 & 1.39 & 336 & 401 \\
5 & $\mathbf{\square}$ & 150 & 1 & 0.150 & 0.20 & 1.39 & 336 & 401 \\
6 & $\bullet$ & 150 & 1 & 0.150 & 0.20 & 1.39 & 336 & 401 \\
\hline \multicolumn{7}{r}{ Remarks: • - cylinder; - cuboid; $\bullet$ - diamond. }
\end{tabular}

\subsection{Effect of Roughness Height}

The roughness height $k$ is one of the most significant parameters to trigger transition behind a disturbance element. Currently used roughness-induced transition methodologies rely upon empirical correlations, mostly based on a critical roughness Reynolds number $\mathrm{Re}_{k k}$, which is built with the roughness height $k$ and the flow values of the unperturbed base flow at that height. Values typically reported in the literature, above which transition already occurs shortly downstream of the roughness, are in the order of $\mathrm{Re}_{k k} \approx 300-500$. Therefore, transition immediately downstream of the roughness is not to be expected for Cases 1-3 from Table 2. However, this criterion does not take into account the roughness shape, the aspect ratio $(k / d)$, the wall temperature, the disturbance environment, and the local pressure gradient. In order to take better into account the wall temperature effect, an alternative definition of roughness Reynolds number $\mathrm{Re}_{k k^{*}}$, recently proposed by Bernardini et al. [25], is added to Table 2, where the dynamic viscosity at the roughness height $k$ is replaced by that at the wall. Depending on the roughness shape and aspect ratio, the critical value of $\operatorname{Re}_{k k^{*}}$ lies between 400 and 700 [25]. The main focus of this work is to study convective wake instabilities. Therefore, no higher roughness elements as listed in Table 2 were investigated, in order to prevent the onset of a 3D global instability, as, for example, recently studied by Loiseau et al. [26].

Figure 7 shows the top view of the near-field flow region around the $k=150 \mu \mathrm{m}$ cylindrical roughness element. Two major flow features are identifiable, which appear in all of the steady solutions that have been investigated within this work (Cases 1-6). The first one is an upstream (see, also, Fig. 8) and downstream reversed flow region (visualized by a black isosurface of zero streamwise velocity) and the second one is a system of vortical structures, which originate from the upstream recirculation zone and wrap around the roughness element. This system exhibits a major counterrotating vortex pair, known as the horseshoe vortex, whose legs bring down high-speed fluid from the outer region of the boundary layer towards the wall and in other parts transport low-speed fluid from the wall to the upper boundary layer region. This transport of mo- 


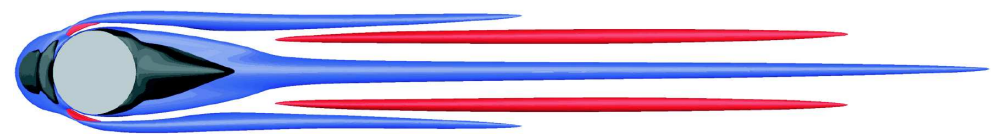

Figure 7 Top view of the flow structures around the $k=150 \mu \mathrm{m}$ cylindrical roughness element: grey color - roughness element; black color — recirculation zone, visualized by an isosurface of zero streamwise velocity; and red/blue color — high- and lowspeed streaks, visualized by isosurfaces of $\Delta u= \pm 0.3$ with $\Delta u$ defined as the difference between normalized streamwise velocity of the base flow with and without roughness element

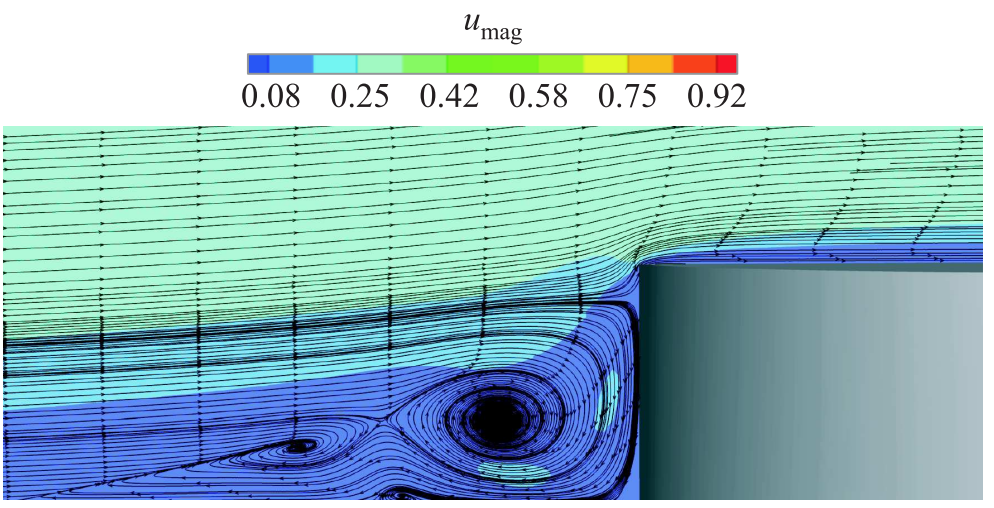

Figure 8 Close-up view of the vortical system upstream of the $k=150 \mu \mathrm{m}$ cylindrical roughness element, highlighted by streamlines in the symmetry plane and colored with the normalized velocity magnitude

mentum is known as the lift-up effect and generates streamwise velocity highand low-speed streaks [27]. The roughness element induces a central low-speed streak due to the streamwise velocity blockage.

Figures 9 and 10 show the influence of increasing roughness height (Cases 14) on the mean streamwise velocity in a plane located two roughness diameters behind the cylindrical roughness trailing edge and its associated shear, expressed through the shear magnitude, $u_{s}$, which is defined as

$$
u_{s}=\sqrt{\left(\frac{\partial u}{\partial y}\right)^{2}+\left(\frac{\partial u}{\partial z}\right)^{2}} .
$$

With increasing roughness height, the central low-speed streak and the vortex system become more pronounced, leading to the formation of the typical mushroom-like structure [28] in the center region with a detached shear layer above the roughness. The footprint of the horseshoe vortex is visible through 


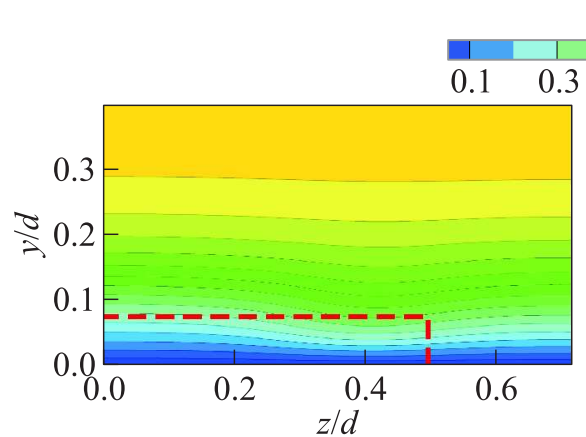

(a)

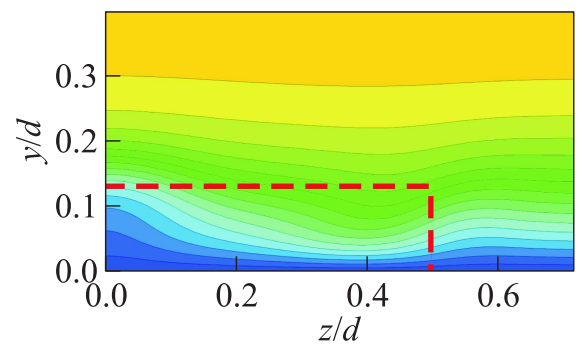

(c)
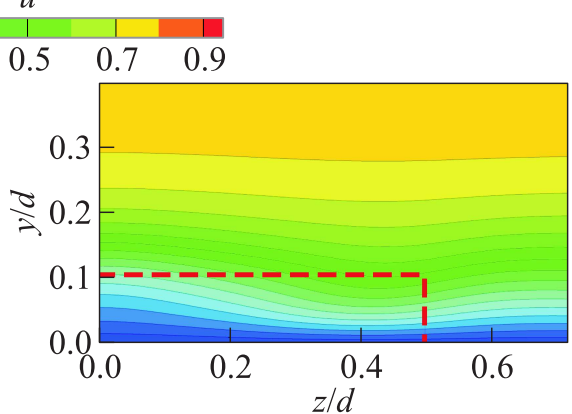

(b)

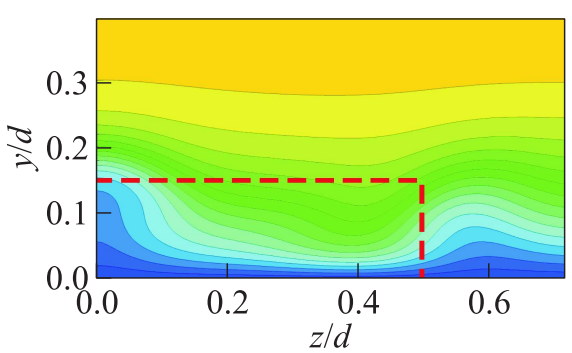

(d)

Figure 9 Contours of streamwise velocity in the roughness wake for 4 different roughness heights at $s=2 \mathrm{~mm}$ for the cylindrical element: (a) $k=75 \mu \mathrm{m}$; (b) 105; (c) 130 ; and $(d) k=150 \mu \mathrm{m}$. Due to the spanwise symmetry of the flowfield, only the right half of the plane is shown. The dashed line indicates the projection of the roughness element

a region of high shear close to the wall. A $2 \mathrm{D}$ spatial instability analysis for the stationary mean flow, generated by the tallest roughness height, in the plane shown in Figs. 9 and 10 reveals both, an amplified symmetric mode (also known as varicose mode) and amplified antisymmetric modes (sinuous mode) with respect to the streamwise velocity fluctuation.

The most amplified symmetric mode, in terms of maximum $N$-factor reached, will be referred to as the dominant symmetric mode (S1) and the most amplified antisymmetric mode will be entitled as the dominant antisymmetric mode (A1), whereas the dominant mode of both mode families ( $\mathrm{S}$ and $\mathrm{A}$ ) will be referred to as the leading mode.

The computed spatial growth rate for modes $\mathrm{S} 1$ and $\mathrm{A} 1$ at $s / d=2$ is given in Fig. 11. Figure 12 shows the normalized mode shapes of the streamwise fluctuation at the disturbance frequency with the highest growth rate. The velocity perturbation of the symmetric mode is concentrated near the top of the 


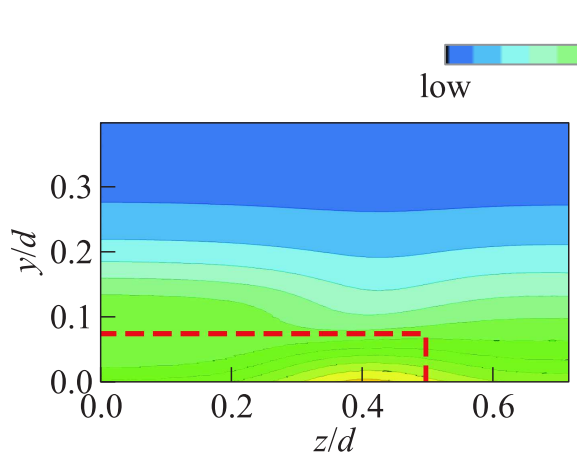

(a)

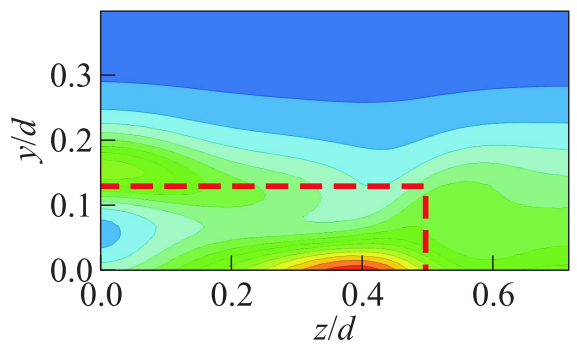

(c)
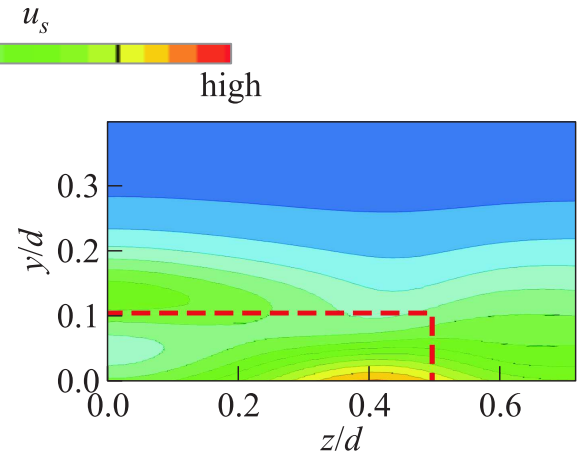

(b)

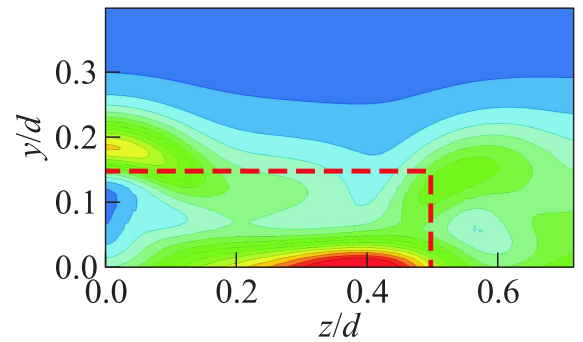

$(d)$

Figure 10 Contours of shear magnitude in the roughness wake for 4 different roughness heights at $s=2 \mathrm{~mm}$ for the cylindrical element: (a) $k=75 \mu \mathrm{m}$; (b) 105; (c) 130; and $(d) k=150 \mu \mathrm{m}$. Due to the spanwise symmetry of the flowfield, only the right half of the plane is shown. The dashed line indicates the projection of the roughness element

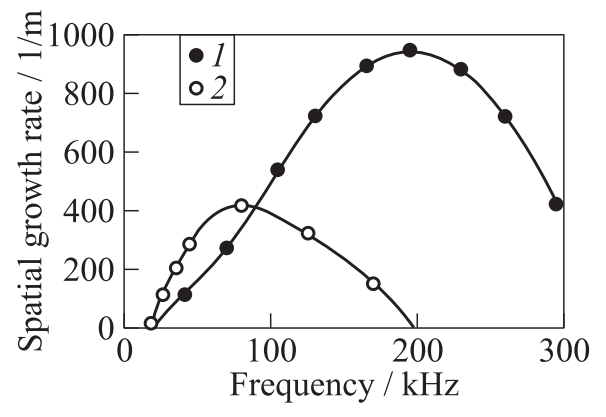

Figure 11 Instability characteristics of the wake flow behind the $k=150 \mu \mathrm{m}$ cylindrical roughness element at $s=2 \mathrm{~mm}$. Spatial growth rate vs. frequency for varicose mode S1 (1) and sinuos mode A1 (2) 


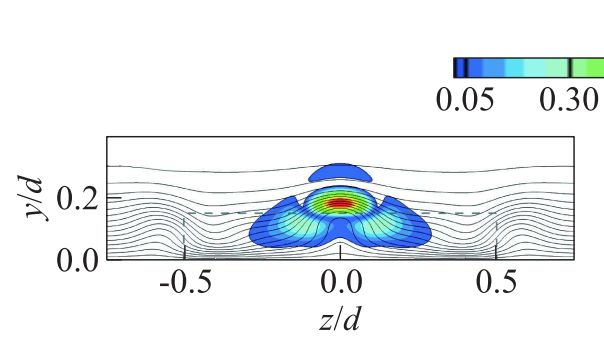

(a)

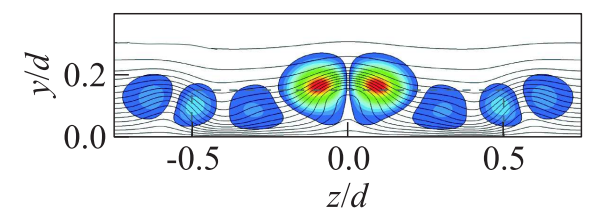

(b)

Figure 12 Instability characteristics of the wake flow behind the $k=150 \mu \mathrm{m}$ cylindrical roughness element at $s=2 \mathrm{~mm}$. Normalized mode shape of streamwise velocity perturbation for modes $\mathrm{S} 1(f=195 \mathrm{kHz})(a)$ and A1 $(f=80 \mathrm{kHz})(b)$. Values $|\hat{u}|<0.05$ are blanked out. The isolines correspond to the basic state $u$-velocity distribution and the dashed line is the projection of the roughness element

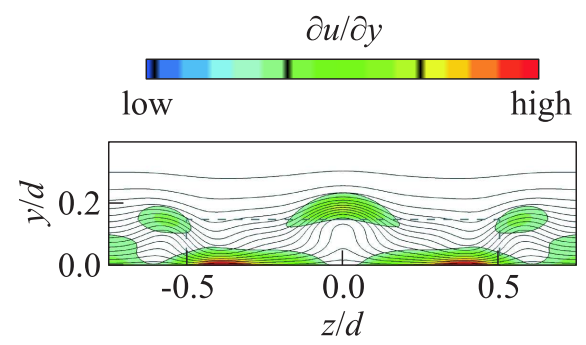

(a)

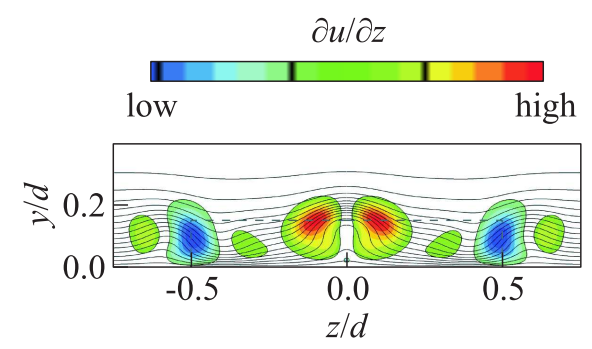

(b)

Figure 13 Instability characteristics of the wake flow behind the $k=150 \mu \mathrm{m}$ cylindrical roughness element at $s=2 \mathrm{~mm}$. Contours of $u$-velocity gradients in wall-normal $(a)$ and spanwise $(b)$ directions. Gradients between $20 \%$ of the maximum and minimum shear are blanked out for the spanwise shear contour plot and values below $27 \%$ of the maximum shear are blanked out for the wall-normal gradient plot. The isolines correspond to the basic state $u$-velocity distribution and the dashed line is the projection of the roughness element

mushroom-like structure in a region with increased wall-normal shear $(\partial u / \partial y)$, as depicted in Fig. 13a. On the other hand, the antisymmetric mode peaks on either side of the mushroom structure, where the spanwise shear $(\partial u / \partial z)$ is large (see Fig. 13b).

In the following, the stability calculations will be extended to the whole wake flow region by tracing the dominant modes downstream at several fixed frequencies. Thereby, the local growth rates will be accumulated to an $N$-factor using Eq. (9). Figure 14 shows the $N$-factor envelope curves vs. the dimensionless 


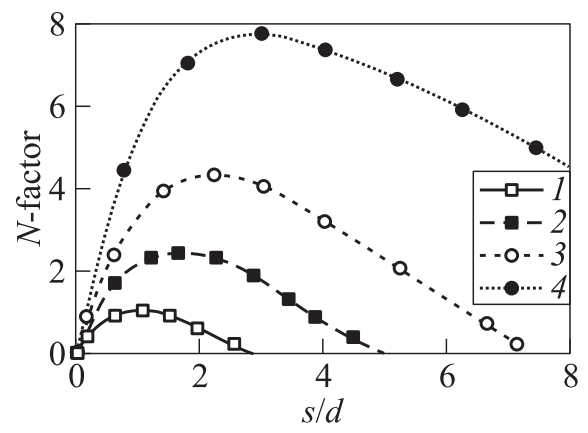

(a)

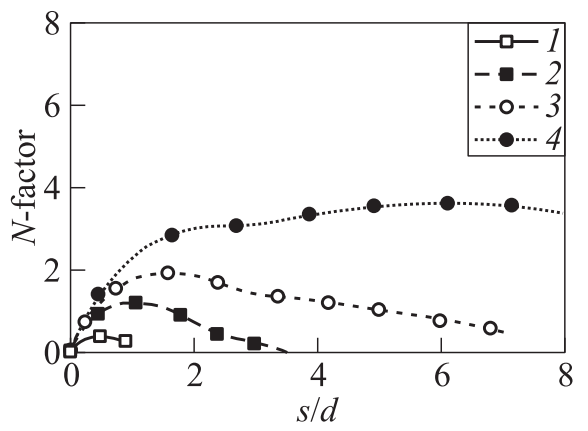

(b)

Figure $14 N$-factor envelope curves vs. surface arc length for the dominant symmetric $(a)$ and antisymmetric $(b)$ modes at 4 different roughness heights: $1-k=75 \mu \mathrm{m}$ and $\operatorname{Re}_{k k}=124 ; 2-k=105 \mu \mathrm{m}$ and $\operatorname{Re}_{k k}=206 ; 3-k=130 \mu \mathrm{m}$ and $\operatorname{Re}_{k k}=278$; and $4-k=150 \mu \mathrm{m}$ and $\operatorname{Re}_{k k}=336$

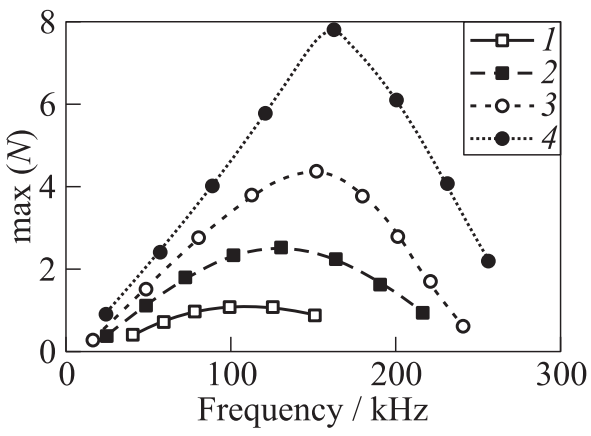

(a)

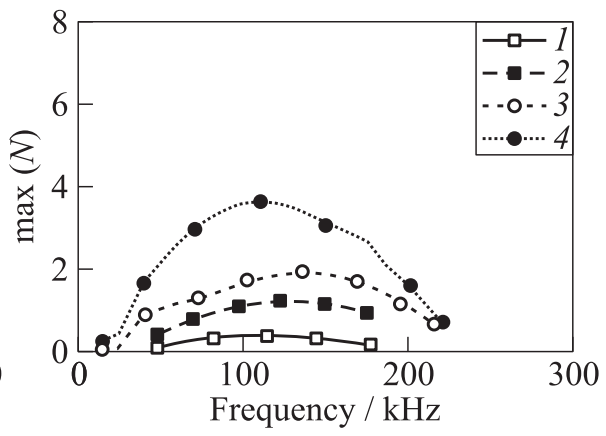

(b)

Figure 15 Maximum $N$-factor obtained downstream of the roughness at the corresponding disturbance frequency: $(a)$ domain symmetric mode; $(b)$ domain antisymmetric mode; $1-k=75 \mu \mathrm{m} ; 2-105 ; 3-130$; and $4-k=150 \mu \mathrm{m}$

surface arc length for modes $\mathrm{S} 1$ and $\mathrm{A} 1$. The location $s / d=0$ denotes the trailing edge of the disturbance element. The actual maximum $N$-factor will be higher than plotted in Figs 14 and 15, because the dominant wake modes are amplified in the direct vicinity of the element (similar observations by Choudhari et al. [28]); thus, a positive growth rate is found at the very first plane for the instability analysis located $25 \mu \mathrm{m}$ downstream of the roughness trailing edge. The $N$-factor increases with roughness height for both dominant modes leading to a maximum $N$-factor for the $\mathrm{S} 1$ and $\mathrm{A} 1$ modes of $N_{\max } \approx 7.8$ and $\approx 3.6$ for $k=150 \mu \mathrm{m}$, respectively. Within this work, the symmetric mode always 
leads to the highest integrated amplification rate, which is in close agreement to the findings of $[12,24,25,28]$ for the near field around the roughness element in a zero pressure gradient flow. The authors of $[12,24,25,28]$ report that depending on the aspect ratio [25], the symmetric mode is the leading mode at first and leads to transition in supercritical cases, where the amplitude of the disturbance caused by the roughness element is sufficient to promote turbulence [29]. However, with increasing distance away from the roughness element, the antisymmetric mode takes over the leading part for subcritical flow conditions. The instability results for the element with the highest roughness Reynolds number $\left(\operatorname{Re}_{k k}=336\right)$ further confirm the reported findings. The dominant varicose mode $\mathrm{S} 1$ leads to the highest $N$-factor and is amplified for about three roughness diameters downstream of the element, whereas the growth rates for the dominant sinuous mode A1 are lower at first, but still increase up to about twice the amplified distance of the symmetric mode. For the lower roughness heights considered in Figs. 14 and 15, the symmetric mode is amplified the furthest downstream.

It can be stated that for the Cases 1-4 from Table 2, the zone of modal amplification is limited to a narrow downstream region behind the protuberance element. The distinct decay of the dominant wake modes is most likely attributed to the strong favourable pressure gradient. Recently, Plogmann et al. [30] experimentally investigated the effect of different pressure gradients on the mode amplitudes behind a cylindrical roughness element. The authors reveal that in the near wake of the roughness $(s / d<4)$, the amplitude of the leading mode is very weakly dependent on the pressure gradient. However, the amplitude in the favourable pressure gradient (FPG) case is slightly lower compared with the zero (ZPG) and adverse pressure (APG) cases. For the far wake region $(s / d>4)$, the authors report that the leading mode is damped in the FPG case, whereas in the ZPG and APG cases, the amplitude remains at a nearly constant level before being slightly damped [30]. A stabilizing influence of the FPG is also mentioned in [9].

Figure 15 shows the maximum $N$-factor, $N_{\max }$, computed downstream of the roughness at its corresponding frequency. Peak disturbance frequencies are higher for mode S1 in comparison to mode A1. This observation is in qualitative agreement to the findings of Choudhari et al. [28]. With increasing roughness height, the maximum $N$-factor is shifted to higher frequencies for the symmetric mode, whereas no clear trend is observable for the dominant antisymmetric mode.

\subsection{Effect of Roughness Planform Shape}

In this subsection, the effect of three different planform shapes (see Fig. 1) on the mean flow and instability results at identical roughness Reynolds numbers are presented. 


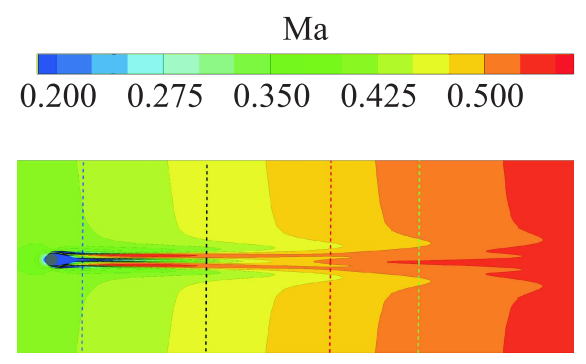

(a)

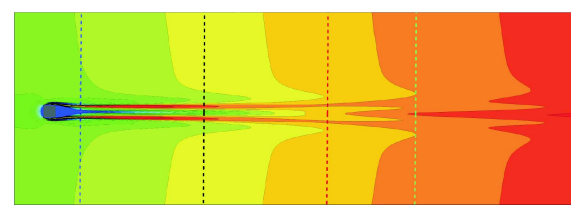

(b)

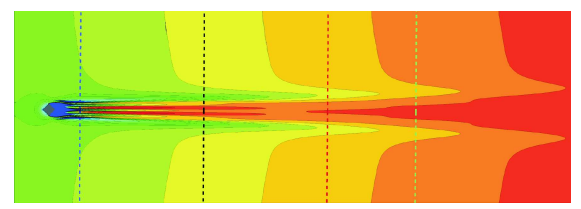

(c)

Figure 16 Mach number distribution at $y=100 \mu \mathrm{m}$ : (a) cylindrical roughness element; $(b)$ cuboidal; and $(c)$ diamond roughness element

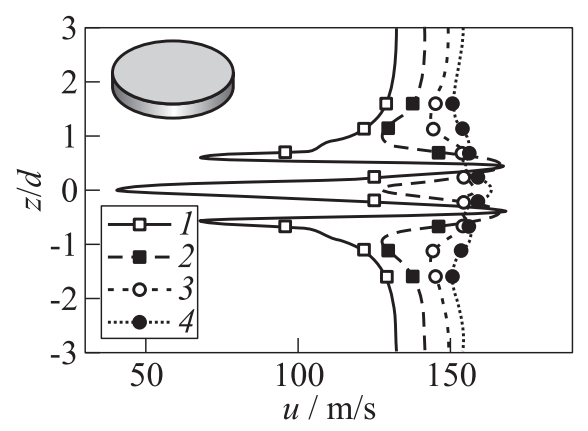

(a)

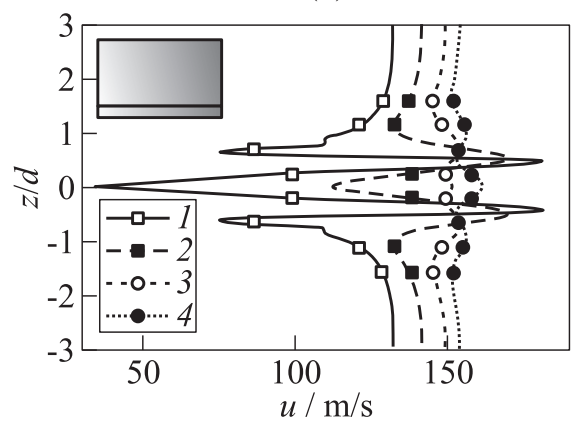

(b)

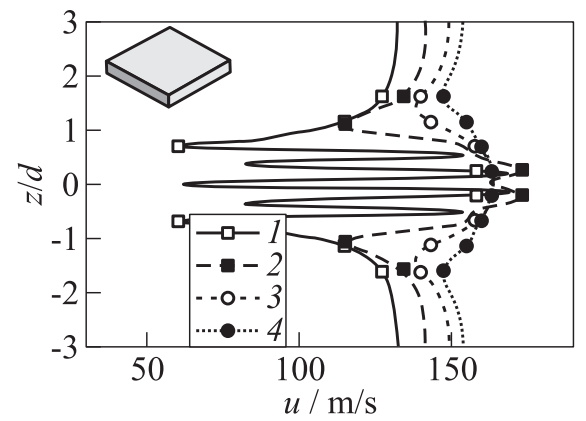

(c)

Figure 17 Wake flow profiles of streamwise velocity at wall normal height $y=100 \mu \mathrm{m}$ for downstream locations: $1-s=2 \mathrm{~mm} ; 2-12 ; 3-23$; and $4-s=30 \mathrm{~mm}$ 
Figure 16 shows isocontours of the Mach number distribution at a constant distance $y=100 \mu \mathrm{m}$ above the capsule surface. The wake flow regions of the cylindrical (see Fig. 16a) and cuboidal (see Fig. 16b) elements exhibit a qualitatively similar amplitude and downstream evolution of the high- and low-speed streaks, whereas the streak pattern behind the diamond roughness element (see Fig. 16c) is different (see, also, Fig. 17). Instead of just one major vortex pair, two pairs of counterrotating vortices are generated by the diamond element. All wake flows have in common that due to the down-wash motion of the vortices (high-momentum fluid from the upper boundary layer part gets pushed towards the wall), the velocity deficit in the central low-speed streak region is first annihilated and then replaced by a high-speed streak. Tumin and Reshotko [31] made similar observations for the flow behind a 3D hump and reported that after the point where the central streak changes its sign, the steady wake flow experiences transient growth. The presence of two counterrotating vortex pairs behind the diamond element accelerates the reduction of the velocity deficit

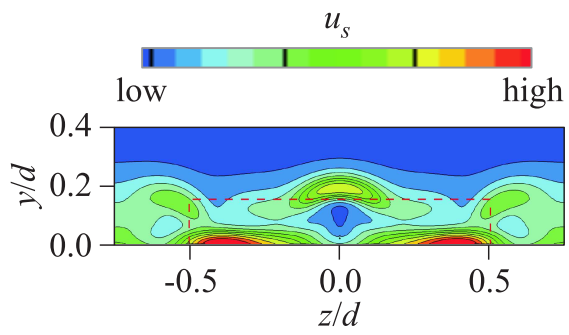

(a)

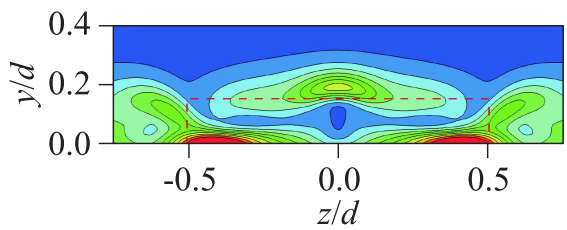

(b)

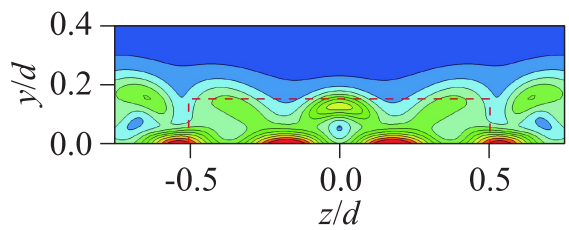

(c)

Figure 18 Contours of shear magnitude in the roughness wake plane at $s=2 \mathrm{~mm}$ : (a) cylindrical roughness element; $(b)$ cuboidal; and $(c)$ diamond roughness element. The dashed line is the projection of the roughness element 
in the wake and forms a stronger and wider central high-speed streak downstream.

Figure 18 depicts the shear magnitude in a plane located two millimeters downstream of the obstacles. In accordance with the wake profiles of streamwise velocity (see Fig. 17), the structure and strength of the shear layers behind the cylinder and cuboid is also very similar, whereas greater differences are observable for the diamond element. Besides the two additional regions of high shear (attributed to the extra vortex pair), the detached shear layer is located closer to the wall, presumably due to the increased down-wash effect induced by the supplemental vortex pair.

The $N$-factor envelopes curves of Cases 4-6 are given in Fig. 19 for modes S1 and A1, respectively. The dominant varicose mode in the wake flow behind the cylindrical roughness generates the highest $N$-factor of all geometries considered; thus, the cylindrical element is the most dangerous obstacle in terms of modal
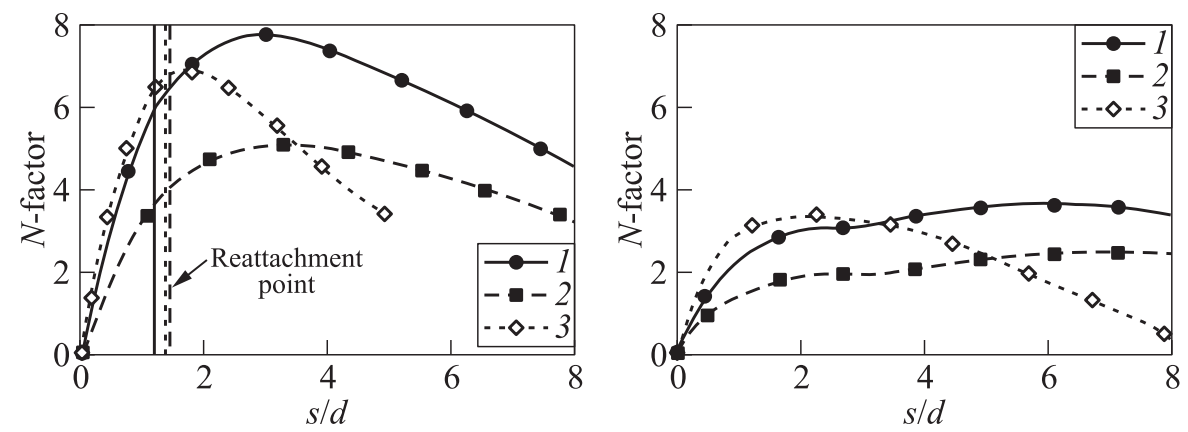

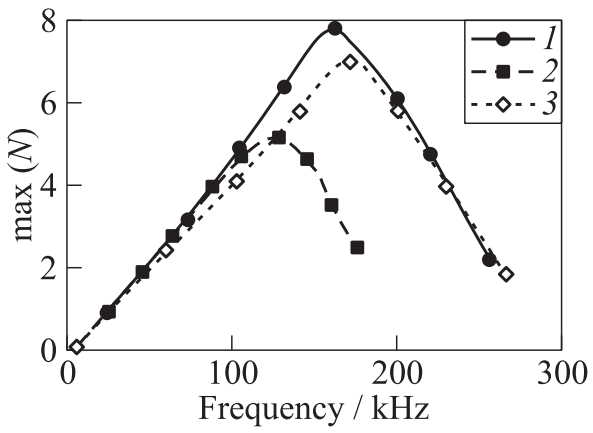

(a)

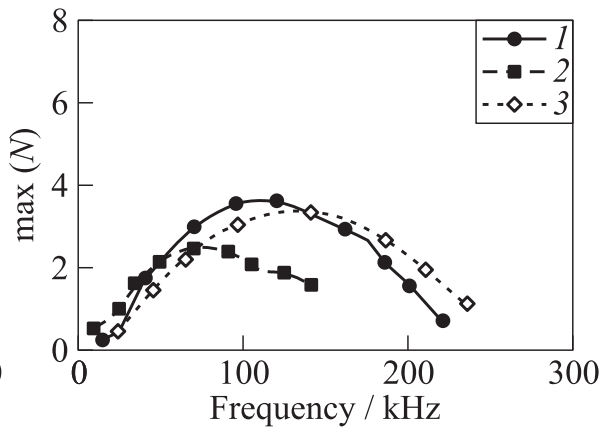

(b)

Figure $19 N$-factor envelope curves vs. surface arc length for the dominant symmetric $(a)$ and antisymmetric modes $(b)$ and maximum $N$-factor obtained downstream of the roughness at the corresponding disturbance frequency for different roughness shapes: 1 - cylinder; 2 - cuboid; and 3 - diamond 
disturbance $N$-factor data, at least for the considered roughness aspect ratio $(k / d)$ and flow conditions. The evolution of the $N$-factor along the nondimensional surface arc length for both mode types of the cuboidal element is very similar to the cylindrical ones, just on a lower level. By regarding the location of the reattachment point in the symmetry plane (vertical line in upper plot of Fig. 19a), it turns out that the region of amplified symmetric disturbances behind the diamond element is limited to the vicinity of the recirculation zone. Peak disturbance frequencies (lower row of plots in Fig. 19) for both mode types decrease with increasing width of the recirculation region.

So far, results have only been presented for the dominant symmetric (S1) and antisymmetric (A1) modes, but the wake flow behind the protuberance element features more amplified modes. Therefore, Fig. 20 shows the spatial growth rate vs. the disturbance frequency of all amplified modes at $s=2 \mathrm{~mm}$. Behind every obstacle, there exists one other amplified antisymmetric mode (A2), which in contrast to mode A1 is amplified in a much narrower frequency band. The maximum growth rate of mode A2 is different for each element; however, the

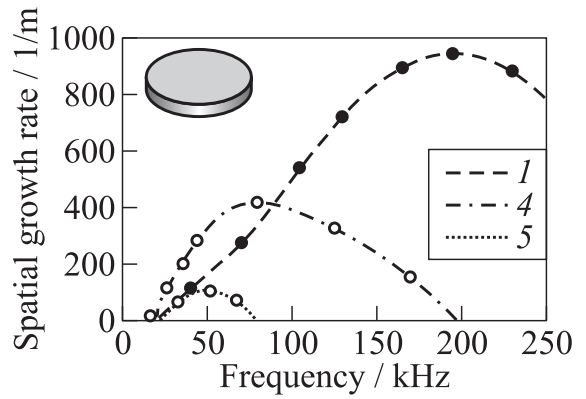

(a)

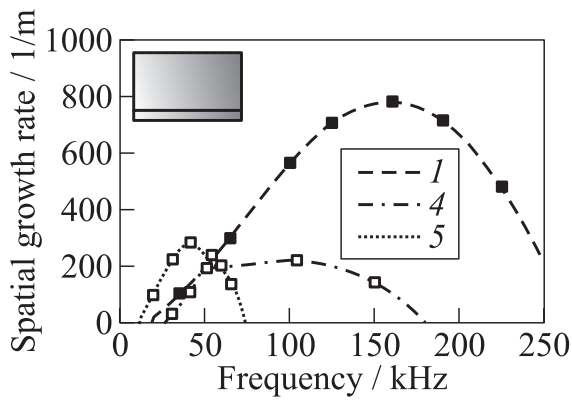

(b)

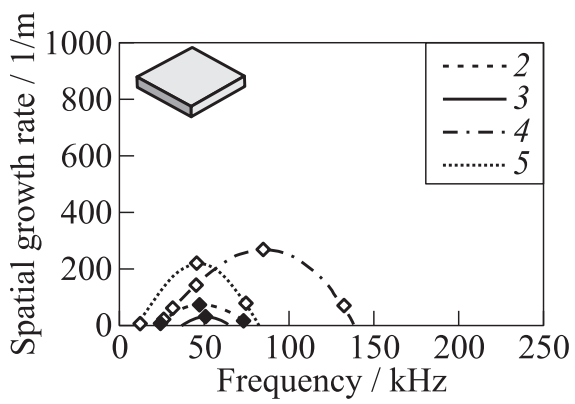

(c)

Figure 20 Spatial growth rate vs. disturbance frequency for all amplified modes at $s=2 \mathrm{~mm}: 1-\mathrm{S} 1 ; 2-\mathrm{S} 2 ; 3-\mathrm{S} 3 ; 4-\mathrm{A} 1 ;$ and $5-\mathrm{A} 2$ 
amplified frequency band is very similar. As indicated by the $N$-factor envelope curves in Fig. 19, the spatial growth rate of mode S1 of the cylindrical element is the highest, whereas mode $\mathrm{S} 1$ is already completely damped at $s=2 \mathrm{~mm}$ behind the diamond element. On the other hand, the wake flow behind the diamond element features two additional amplified symmetric modes (S2 and S3), which are not amplified behind the other two geometries at the considered position.

Figures 21-23 provide an overview of the normalized mode shapes of streamwise velocity fluctuation for modes $\mathrm{S} 1, \mathrm{~A} 1$, and $\mathrm{A} 2$ at $s=2 \mathrm{~mm}$ at the cor-

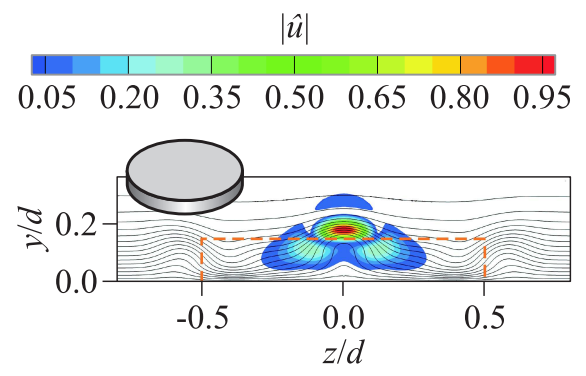

(a)

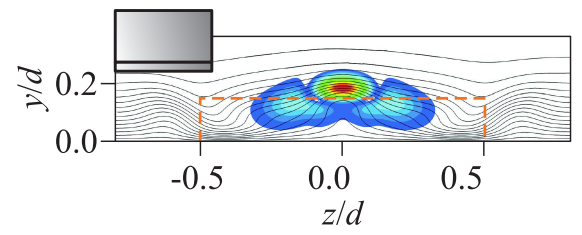

(b)

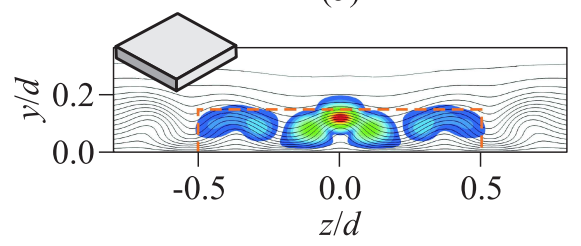

(c)

Figure 21 Normalized mode shapes of streamwise velocity fluctuation at $s=2 \mathrm{~mm}$ for mode S1: $(a) f=195 \mathrm{kHz}$; (b) 160; and (c) $f=173 \mathrm{kHz}$. The black isolines indicate the basic state $u$-velocity distribution and the dashed line represents the projection of the roughness element. Values $|\hat{u}|<0.05$ are blanked out

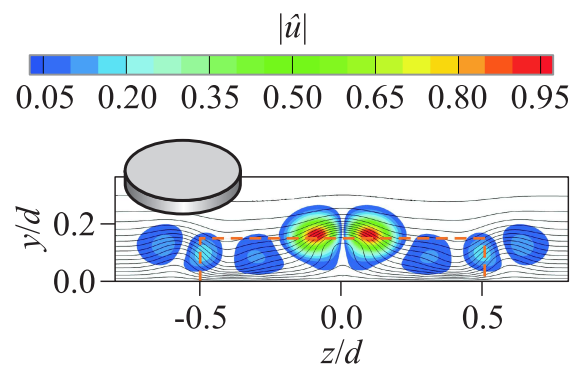

(a)

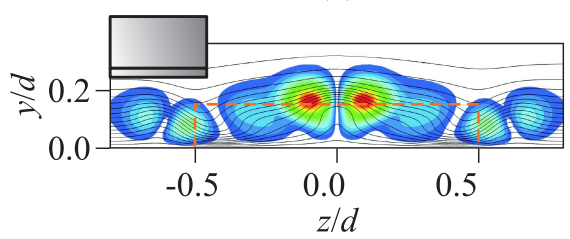

(b)

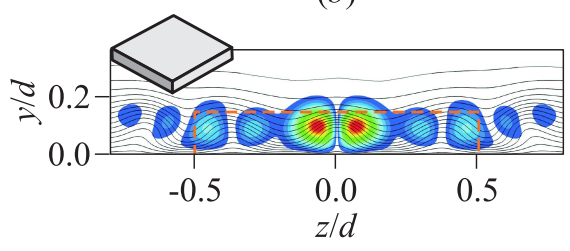

(c)

Figure 22 Normalized mode shapes of streamwise velocity fluctuation at $s=2 \mathrm{~mm}$ for mode A1: $(a) f=80 \mathrm{kHz}$; (b) 105 ; and (c) $f=85 \mathrm{kHz}$. The black isolines indicate the basic state $u$-velocity distribution and the dashed line represents the projection of the roughness element. Values $|\hat{u}|<0.05$ are blanked out 
responding peak disturbance frequency from Fig. 20. In addition, the mode shape of mode $\mathrm{S} 1$ for the diamond element is also given for completeness, despite being damped already. Velocity fluctuations of mode S1 are concentrated near the top of the detached shear layer (see, also, Fig. 18), whereas the sinuous modes A1 and A2 peak on either side of the mushroom-like structure. The mode shape, as well as the peak disturbance frequency, is very similar for each of the amplified mode families shown in Figs. 21-23. Figure 24 depicts the mode shape comparison of modes S1-S3 behind the diamond element at

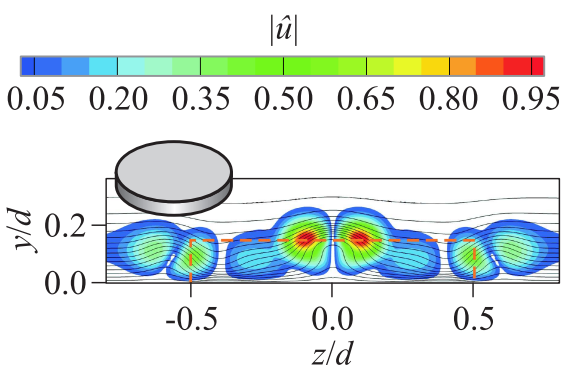

(a)

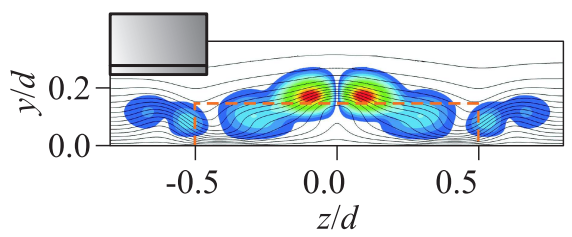

(b)

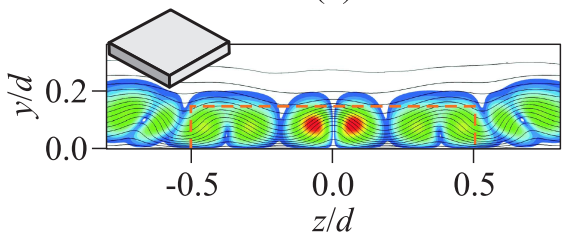

(c)

Figure 23 Normalized mode shapes of streamwise velocity fluctuation at $s=2 \mathrm{~mm}$ for mode A2: (a) $f=50 \mathrm{kHz}$; (b) 42 ; and (c) $f=46 \mathrm{kHz}$. The black isolines indicate the basic state $u$-velocity distribution and the dashed line represents the projection of the roughness element. Values $|\hat{u}|<0.05$ are blanked out

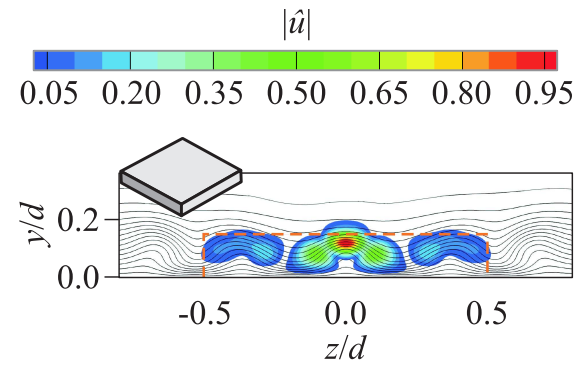

(a)

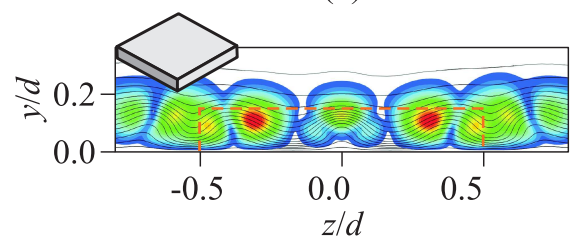

(b)

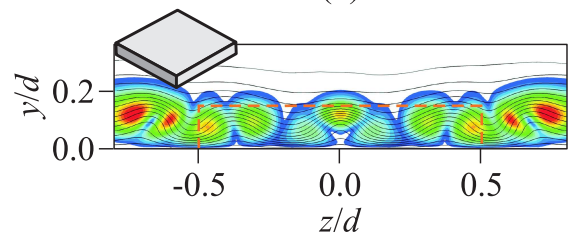

(c)

Figure 24 Normalized mode shapes of streamwise velocity fluctuation at $s=2 \mathrm{~mm}$ for modes $\mathrm{S} 1$ $(f=173 \mathrm{kHz})(a), \mathrm{S} 2(f=48 \mathrm{kHz})(b)$, and $\mathrm{S} 3(f=51 \mathrm{kHz})(c)$. The black isolines indicate the basic state $u$-velocity distribution and the dashed line represents the projection of the roughness element. Values $|\hat{u}|<0.05$ are blanked out 
$s=2 \mathrm{~mm}$. The velocity fluctuations of the higher modes (S2 and S3) are no longer concentrated around the detached shear layer, but rather around the inner vortex pair (mode S2) and outer vortex pair (S3), indicating different mode families.

In this work, a relationship between the spatial growth rate and the streak amplitude (Eq. (7)) is observed.

Figure 25 shows the spatial growth rate at three downstream locations behind the protuberance elements vs. the streak amplitude for all flow cases listed in Table 2. Those plots include additional results from a roughness edge length variation, which are not discussed in further detail in the present paper. The growth rate plotted is the maximum growth rate obtained from all modes over all frequencies. With increasing distance from the obstacle trailing edge, a linear dependency seems to evolve, indicated by the dashed trend line. The observed linear-like trend is in qualitative agreement to the findings of Andersson et al. [32] and Van den Eynde and Sandham [33].

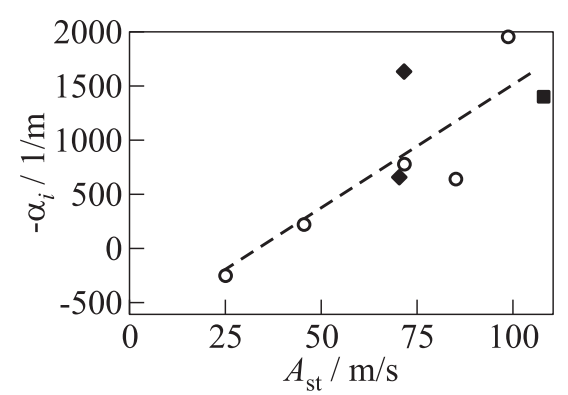

(a)

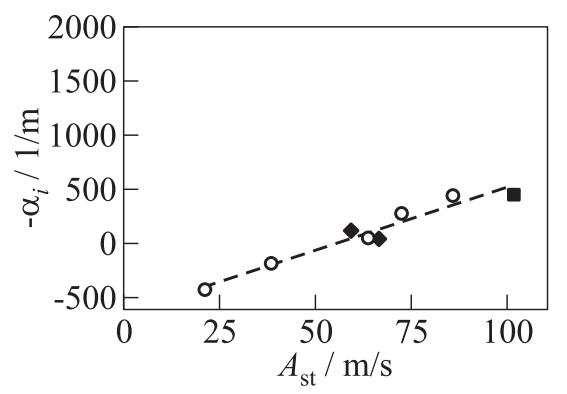

(b)

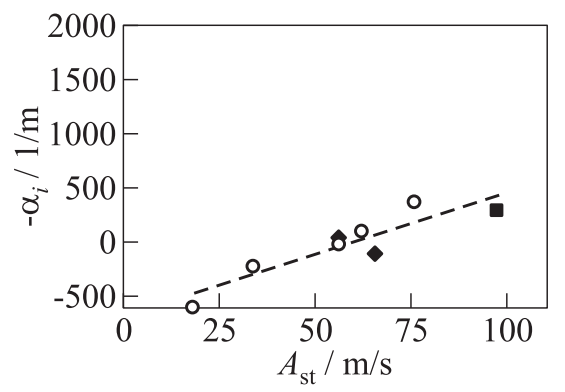

(c)

Figure 25 Maximum spatial growth rate vs. streak amplitude at three downstream locations: (a) $s=1.5 \mathrm{~mm}$; (b) 2.5 ; and (c) $s=3.5 \mathrm{~mm}$. The symbols represent the different flow cases and the dashed line is the best-fit in a linear least squares sense 


\section{CONCLUDING REMARKS}

Laminar stationary base flow computations have been performed for a roughness element submerged in the boundary layer along the leeward symmetry plane of an Apollo-shaped, generic reentry capsule at cold hypersonic freestream conditions at $\mathrm{AOA}=24^{\circ}$. The instability properties of the streaky wake flow behind a cylindrical protuberance element at various heights have been investigated with the help of spatial 2D instability theory. The roughness element induces a central low-speed streak, due to the streamwise velocity blockage, in whose vicinity the streamwise velocity fluctuations of the most amplified symmetric (also known as varicose) and antisymmetric (sinuous) modes are concentrated. The effect of different planform shapes on the mean flow and instability characteristics have been studied by additionally investigating the wake flow behind a diamond and cuboidal elements. It turned out that the symmetric mode always led to the highest maximum $N$-factor downstream of the element, even though in the highest roughness Reynolds number case considered $\left(\operatorname{Re}_{k k}=336\right)$, the antisymmetric mode is amplified over a longer downstream range. The cylindrical geometry is the most dangerous one in terms of maximum disturbance $N$-factor reached, at least for the roughness aspect ratio and flow conditions considered in this work.

The region of amplified disturbances is limited to a few roughness diameters downstream of the protuberance element, presumably due to the strong favourable pressure gradient in the boundary layer of the Apollo capsule. In virtue of the downstream recirculation zone in that region and the formation of streamwise vortices downstream, the consequences of the local parallel flow assumption made for the $2 \mathrm{D}$ spatial stability analysis may be significant. Therefore, future instability analyses should be performed by including the wall-normal velocity in the $2 \mathrm{D}$ eigenvalue computation or by using a PSE-3D method, which also takes into account the streamwise gradients. Furthermore, transient growth should also be considered as a possible source for transition behind a roughness element.

A linear-like trend has been observed between the spatial growth rate and the streak amplitude at fixed downstream locations for all investigated flow cases. Nevertheless, additional parameter combinations, like the wall temperature influence or the presence of a 3D boundary layer at the roughness location, have to be considered in order to further test this trend.

\section{ACKNOWLEDGMENTS}

The authors would like to thank Dr. Pedro Paredes for providing the base flow and instability results of the validation case and Dr. Heinrich Lüdeke for the 
support and extensions to the FLOWer code. The authors also gratefully acknowledge funding of this work by the German Research Foundation (DFG) grant HE 5440/4-1 within the project HYPTRANS (PAK 742).

\section{REFERENCES}

1. Hollis, B. R. 2012. Blunt-body entry vehicle aerothermodynamics: Transition and turbulent heating. J. Spacecr. Rockets 49(3):435-449.

2. Reshotko, E. 2008. Transition issues for atmospheric entry. J. Spacecr. Rockets 45(2):161-164.

3. Johnson, H.B., G. V. Candler, and M. J. Wright. 2006. Boundary layer stability analysis of Mars Science Laboratory aeroshell. AIAA Paper No. 2006-920.

4. Chang, C.-L., M. Choudhari, B. R. Hollis, and F. Li. 2009. Transition analysis for the Mars Science Laboratory entry vehicle. AIAA Paper No. 2009-4076.

5. Theiss, A., S. Hein, D. Heitmann, S. R. C. Ali, and R. Radespiel. 2014. Numerical and experimental investigation of laminar-turbulent boundary layer transition on a blunt generic re-entry capsule. AIAA Paper No. 2014-2353.

6. Li, F., M. Choudhari, C.-L. Chang, and J. White. 2011. Boundary layer transition over blunt hypersonic vehicles including effects of ablation-induced out-gassing. AIAA Paper No. 2011-3303.

7. Hollis, B. R. 2014. Distributed roughness effects on blunt-body transition and turbulent heating. AIAA Paper No. 2014-0238.

8. Amar, A. J., T. J. Horvath, B. R. Hollis, K. T. Berger, S. A. Berry, and N. Calvert. 2008. Protuberance boundary layer transition for project Orion Crew Entry Vehicle. AIAA Paper No. 2008-1227.

9. Chang, C.-L., M. Choudhari, F. Li, and B. Venkatachari. 2011. Effects of cavities and protuberances on transition over hypersonic vehicles. AIAA Paper No. 20113245 .

10. Schneider, S. P. 2008. Hypersonic boundary-layer transition on blunt bodies with roughness. AIAA Paper No. 2008-501.

11. Reda, D. C. 2002. Review and synthesis of roughness-dominated transition correlations for reentry applications. J. Spacecr. Rockets 39(2):161-167.

12. Groskopf, G., M. J. Kloker, and O. Marxen. 2008. Bi-global secondary stability theory for high-speed boundary layer flows. CTR Summer Program 2008 Proceedings $55-72$,

13. Kegerise, M. A., R. A. King, M. Choudhari, F. Li, and A. Norris. 2014. An experimental study of roughness-induced instabilities in a supersonic boundary layer. AIAA Paper No. 2014-2501.

14. Bridges, T., and P. Morris. 1984. Differential eigenvalue problems in which the parameter appears nonlinearly. J. Comput. Phys. 55:437-460.

15. Raddatz, J., and J.K. Fassbender. 2005. Block structured Navier-Stokes solver FLOWer. MEGAFLOW - numerical flow simulation for aircraft design. Eds. 
N. Kroll and J.K. Fassbender. Notes on numerical fluid mechanics and multidisciplinary design ser. 89:27-44.

16. Balay, S., S. Abhyankar, M. Adams, J. Brown, P. Brune, K. Buschelman, V. Eijkhout, W. Gropp, D. Kaushik, M. Knepley, L. C. McInnes, K. Rupp, B. Smith, and H. Zhang. 2014. PETSc Users manual. Technical report ANL-95/11 - revision 3.5. Argonne National Laboratory.

17. Hernandez, V., J.E. Roman, and V. Vidal. 2005. SLEPc: A scalable and flexible toolkit for the solution of eigenvalue problems. ACM Trans. Math. Software 31(3):351-362.

18. Maschhoff, K. J., and D. C. Sorensen. 1996. PARPACK: An efficient portable large scale eigenvalue package for distributed memory parallel architectures. Applied parallel computing. Industrial computation and optimization. Eds. J. Wasniewski, J. Dongarra, K. Madsen, and D. Olesen. Lecture notes in computer science ser. 1184:478-486.

19. Lehoucq, R. B., D. C. Sorensen, and C. Yang. 1998. ARPACK users guide. Solution of large-scale eigenvalue problems by implicitly restarted Arnoldi methods. Philadelphia, PA: SIAM.

20. Amestoy, P., I. S. Duff, J.-Y. L'Excellent, and J. Koster. 2001. A fully asynchronous multifrontal solver using distributed dynamic scheduling. SIAM J. Matrix Anal. Appl. 23(1):15-41.

21. Hermanns, M., and J. A. Hernández. 2008. Stable high-order finite-difference methods based on non-uniform grid point distributions. Int. J. Numer. Meth. Fluids 56:233-255.

22. Paredes, P., M. Hermanns, S. Le Clainche, and V. Theofilis. 2013. Order $10^{4}$ speedup in global linear instability analysis using matrix formation. Comput. Meth. Appl. Mech. Eng. 253:287-304.

23. Paredes, P., V. Theofilis, D. Rodríguez, and J. A. Tendero. 2011. The PSE-3D instability analysis methodology for flows depending strongly on two and weakly on the third spatial dimension. AIAA Paper No. 2011-3752.

24. De Tullio, N., P. Paredes, N.D. Sandham, and V. Theofilis. 2013. Laminarturbulent transition induced by a discrete roughness element in a supersonic boundary layer. J. Fluid Mech. 735:613-646.

25. Bernardini, M., S. Pirozzoli, P. Orlandi, and S. K. Lele. 2012. Compressible boundary layer transition induced by isolated roughness elements. CTR Summer Program 2012 Proceedings. 15-24.

26. Loiseau, J.-C., J.-C. Robinet, S. Cherubini, and E. Leriche. 2014. Investigation of the roughness-induced transition: Global stability analyses and direct numerical simulations. J. Fluid Mech. 740:175-211.

27. Landhal, M. T. 1990. On sublayer streaks. J. Fluid Mech. 212:593-614.

28. Choudhari, M., F. Li, W. Minwei, C. L. Chang, and J. Edwards. 2010. Laminarturbulent transition behind discrete roughness elements in a high-speed boundary layer. AIAA Paper No. 2010-1575.

29. Bernardini, M., S. Pirozzoli, P. Orlandi, and S. K. Lele. 2014. Parameterization of boundary-layer transition induced by isolated roughness elements. AIAA J. 52(10):2261-2269. 
30. Plogmann, B., W. Würz, and E. Krämer. 2014. On the disturbance evolution downstream of a cylindrical roughness element. J. Fluid Mech. 758:238-286.

31. Tumin, A., and E. Reshotko. 2004. The problem of boundary-layer flow encountering a three-dimensional hump revisited. AIAA Paper No. 2004-0101.

32. Andersson, P., L. Brandt, A. Bottaro, and D. Henningson. 2001. On the breakdown of boundary layer streaks. J. Fluid Mech. 428:29-60.

33. Van den Eynde, J. P. J. P., and N. D. Sandham. 2014. Numerical simulation of roughness-induced instability growth and transition at Mach 6. AIAA Paper No. 2014-2499. 